\title{
A Preliminary Study of 316L Stainless Steel Finishing Process by the Tumbling Method with Acrylic Abrasive Media
}

${ }^{1}$ F.P. Putera

${ }^{2}$ Suyitno

1Department of

Mechanical and

Industrial Engineering, Faculty of Engineering, Universitas Gadjah Mada. Jl. Grafika 2, Yogyakarta 55281, Indonesia

${ }^{2}$ Centre for Innovation of Medical Equipment and Devices

(CIMEDs), Faculty of Engineering, Universitas Gadjah Mada, Yogyakarta 55281, Indonesia

Email:

1finnyputera@gmail.com

\section{Keywords}

hip joint prosthesis, tumble finishing, surface roughness.

\begin{abstract}
A certain level of roughness of the surface of a hip prosthesis is necessary to minimize wear on components. Smoothing of the surface roughness can be performed by the tumble finishing method. This research was aimed to figure out the effect of spindle rotary speed on the level of roughness of the test specimen surface. The test specimen was made of 316 stainless steel, which is one of the materials constituting the component of the femoral head in a hip prosthesis. The abrasive media used was acrylic type plastic with a 4-mm-diameter spherical profile. The results of this tumbling process experiment demonstrate that the effective speed at which the cover surface of the test specimen was smoothed was the spindle rotary speed of $125 \mathrm{rpm}$.
\end{abstract}




\section{Introduction}

Degradation of hip joint function in medical terminology is referred to as hip osteoarthritis disease. In those suffering from osteoarthritis disease, the cartilage in the joint undergoes wear or thinning due to fraction (Cahyono, 2011). This wear or thinning will cause the surface of the hip joint cartilage corrugated and uneven. Aside from pain, hip osteoarthritis causes the hip joint to have disturbed motion, occasionally with noise, and even dislocation from the normal position (Cahyono, 2011).

Hip osteoarthritis can be overcome in a variety of ways, one of which is hip replacement with a hip prosthesis fitted into the body through surgical procedures. To create a good prosthetic hip joint, the component surfaces must be smooth so that the remaining contact stresses can be minimized (Chowdury et al., 2004). The smoothing process of the component surfaces in an artificial knee can be undertaken by deburring and finishing (Barletta et al., 2014).

Tumble finishing is suited for smoothing the femoral head component of hip joint prosthesis, which consists of internal and external surfaces. It is a high-efficiency, low-cost surface smoothing process of high efficiency with fine smoothing outcomes (Yang et al., 2017).

Tumble finishing involves rotation of a barrel containing abrasive media with a part immersed within, removing the coarse particles from machining on the internal and external surfaces of the part and leading to improved surface smoothness (Yang et al., 2017).

Figure 1 depicts the scheme for a tumble finishing process. In this process, the part and the abrasive media are placed in a barrel (container). When the barrel rotates at a predetermined speed, the part and the abrasive media will be lifted in the direction of the barrel rotation with influences from centrifugal, gravitational, and frictional forces. On the onset, the part and the abrasive media are positioned at the bottom of the barrel in an equilibrium, relatively motionless. When the part and the abrasive media are on the upper area of the barrel at a given height, the two objects will slide down. Then, the abrasive and part will tumble and initiate an abrasive process in the part.

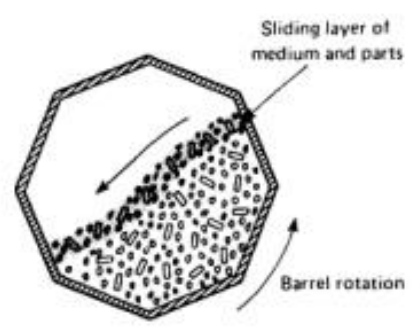

Figure 1. Tumbling scheme (Cotell et al., 1994)

A study pertaining to tumble finishing was carried out in a 2 -mm-diameter spherical capsule in a $60-\mathrm{mm}$-long, 25-mmdiameter acrylic cylindrical container (Suratwala, 2012). The tumbling process lasted for 96 hours at a rotary speed of 100 $\mathrm{rpm}$. The abrasive media used was glass with a silica colloid serving as a lubricant. It was concluded that this process successfully smoothened the surface of choice from an initial surface of $20 \mu \mathrm{m}$ to $10 \mu \mathrm{m}$.

In the present study, the abrasive media used was plastic. A plastic abrasive media can produce a smooth surface faster than a ceramic one (Yang et al., 2017). A turning machine of which the rotating spindle is modified can be used as a tumbling machine. The quite high purchasing or fabricating cost for a tumble finishing machine led this research to using a turning machine with a modified rotating spindle. Althouh using a cheaper machine, we hoped that the surface roughness still can be decreased by using the tumble process. To reach it in this study, we examined an optimal paramaterto achieve the least rouhness when using the cheaper machine. 


\section{Methodology}

Before tumble finishing is procesed, A barrel is manufactured. A barrel is a container where a test specimen is placed in a turning machine. The barrel in this study was made of 201 SS in a cylindrical form with covering $6 \mathrm{~mm}$ thick. The container was 164 $\mathrm{mm}$ in external diameter and $160 \mathrm{~mm}$ in height. The size was adjusted to the space within the turning machi ne. The design of the barrel must meet the wear-resistance criteria as the wall of the barrel would often rub on the test specimen and the abrasive media.

The abrasive media used in this tumble finishing process was an acrylic type plastic media. The acrylic used had a spherical profile $6 \mathrm{~mm}$ in diameter. A plastic abrasive media is reliable during deburring, ball burnishing, drying, and dry polishing processes (Holzknecht, 2009). The abrasive media used was then placed into a $36-\mathrm{cm}$ diameter stainless steel barrel (201 SS). The amount of the abrasive media was $5 \mathrm{~kg}$. The abrasive media used in this tumble finishing was $6 \mathrm{~mm}$ deep acrylic type plastic. This acrylic type was available in jewelry-accessory stores because its initial designation is for jewelry beads. Standard abrasive media for finishing applications was not readily available in Indonesia.

The specimen used was a solid cylindrical specimen with a hole giving it an internal surface, shaped like a femoral head. This specimen was made of a 316L stainless steel material. The dimensions were $16 \mathrm{~mm}$ in diameter and $25 \mathrm{~mm}$ in length. These material selection and geometrical determination were performed according to the material and the geometrical shape of the hip prosthesis femoral head as shown in Figure 2.

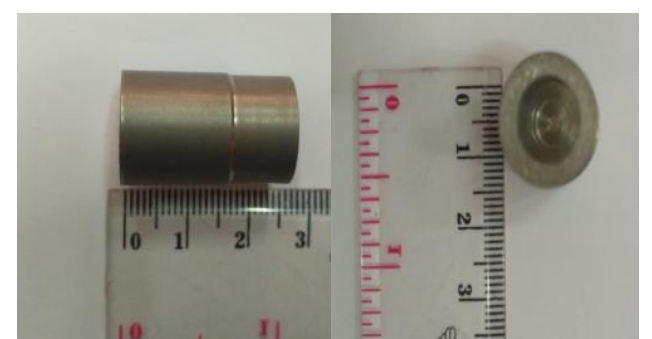

Figure 2. Specimen

Prior to the start of the tumble finishing in the femoral head component, tumble finishing was first performed on the specimen to minimize the frequency of femoral head component use in this research out of high cost of femoral head component.

The test specimen shown in Figure 2 was manufactured through processes from cutting, to drilling, to surface lathing until the desired size was achieved. Then, surface lathing was performed to feed the specimen surface to obtain uniformity in the initial surface roughness measure of the specimen. This process was conducted using a $\mathrm{CNC}$ lathe machine with feeding $0.4 \mathrm{~mm}$ deep, resulting in $0.704 \mu \mathrm{mRa}$ of mean initial surface roughness of covering.

After the barrel, abrasive media and test specimen were made available, the tumble finishing was started. The tumble finishing process is carried out at various spindle speeds (40, 85, 125 and $180 \mathrm{rpm})$ with the same number of turns of 2400 turns.

During the tumbling process, the barrel was rotated by the turning machine spindle, causing the abrasive media along with the part within the barrel to rotate. The speed that results in the lowest surface roughness change then used to test the effect of finishing duration on roughness surface. The duration used varies $(10,20,30,60$ minutes) until there is no change in $\mathrm{Ra}>0.01 \mu \mathrm{m}$. In each variation the surface roughness of the test specimens is measured.

The measurement of the initial and final surface roughness of the test specimen produced from surface lathing was performed 
using the stylus profilometer surface roughness measuring instrument. Part of specimen measured as shown ini Figure 3. The surface roughness is measured three times on different specimens.
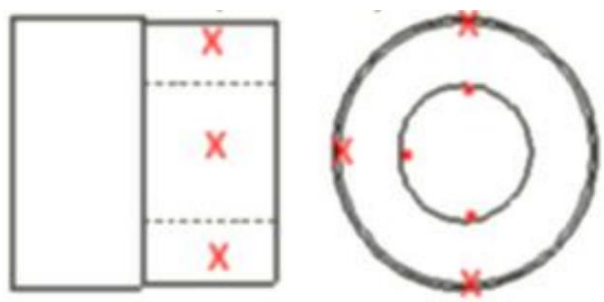

Figure 3. Part of specimen measured

\section{Results}

The outcomes of the tumble finishing process with the spindle rotary speed made variable with surface roughness can be seen in Figure 4. The value of surface roughness was measured with mean (filled circle) and standard error (errorbar).

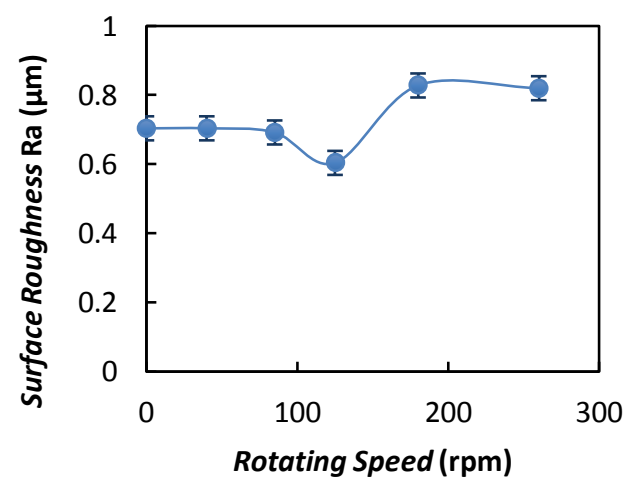

Figure 4. Chart of the effect of rotary speed (rpm) on surface roughness

Based on the chart in Figure 3 , the lowest roughness value obtained was 0.604 $\mu \mathrm{mRa}$. At first, the surface roughness values barely changed from $40 \mathrm{rpm}$ to $85 \mathrm{rpm}$ speeds, but a decline was observed at $125 \mathrm{rpm}$. This means that at $125 \mathrm{rpm}$, the finishing process was running effectively in the tumbling because the increased rotation speed was followed with increased centrifugal force, causing the finishing process to start in the barrel (Yang, 2006). When the rotary speed increased, the part and the abrasive media would also rotate (roll) faster and slide down more often.

However, the surface roughness increased even further at 180 and $260 \mathrm{rpm}$. This was estimated to happen because the tumbling process had passed the effective maximum speed, so the part and the abrasive would cling to the barrel wall during the tumbling process.

After getting the best speed at 125 $\mathrm{rpm}$, then followed by the tumbling process using that speed with vary the duration. The value of surface roughness can be seen in Figure 5. The value of surface roughness was measured with mean (filled circle) and standard error (errorbar).

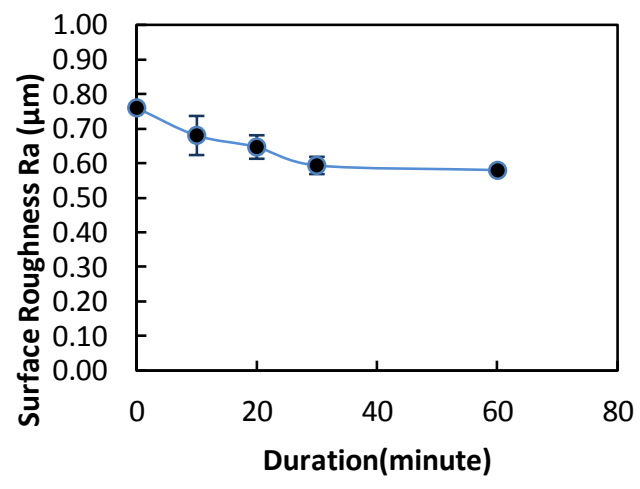

Figure 5. Chart of the effect of duration (minute) on surface roughness

The Figure 4 shows that an increase in finishing duration will causes a decrease in surface roughness to the point of saturation where an increase in duration no longer causes a decrease in roughness significant. Significant decrease in surface roughness still occurs when the duration is 30 minutes which results in surface roughness of $0.59 \mu \mathrm{m}$. After increasing the duration by more than 30 
minutes, the decrease in surface roughness did not occur significantly.

\section{Conclusion}

Finishing process in 316L SS as a hip prosthesis material can be performed through a tumble finishing process with a 6-mmdiameter spherical acrylic abrasive media. This tumble finishing used a turning machine to rotate a barrel containing an abrasive media and a test specimen. From the finishing processes conducted, it was found that the effective maximum rotary speed at which the lowest surface roughness was obtained was $125 \mathrm{rpm}$.

\section{References}

[1] Barletta, M., Pietrobono, F., Rubino, G., Tagliaferri, V., 2014, Drag finishing of sensitive parts with fluidized abrasives, Journal of Manufacturing Processes, Volume 16, pages 494-502.

[2] Cahyono, I., 2011, Simulasi Kontak Komponen Acetabular pada Sambungan Tulang Pinggul Buatan Menggunakan Metode Elemen Hingga, Universitas Dipenogoro.

[3] Cotell, C.M., Sprague, J.A., Smidt, F.A, 1994, Surface Engineering,ASM Handbook, Volume 5

[4] Chowdury, S.K.R., Mishra, A., Pradhan, B., dan Saha, D., 2004, Wear Characteristic and biocompattibility of some polymer composite acetabular cups, Wear, Volume 256, Pages 1026-1036

[5] Holzknecht, E., 2009, Everything you need to know about mechanical/mass finishing: a workshop on the role of media in mechanical surface finishing, Metal Finishing, Volume 107, Pages 27 31.

[6] Suratwala, W.A., Steele, M.D., Feit, K., Moreno, M., Stadermann, J., Fair, K., Chen, A., Nikroo,K., Youngblood, K., 2012, Polishing and local planarization of plastic spherical capsules using tumble finishing, Applied Surface Science 261 Pages 679-689

[7] Yang, S., Li, S.W., 2017, Surface finishing theory and new technology, 1st ed., Springer, Beijing. 